\title{
Intraretinal hyperreflective foci on spectral- domain optical coherence tomographic images of patients with retinitis pigmentosa
}

This article was published in the following Dove Press journal:

Clinical Ophthalmology

24 February 2014

Number of times this article has been viewed

Masako Kuroda'

Yasuhiko Hirami ${ }^{1-3}$

Masayuki Hata ${ }^{4}$

Michiko Mandai ${ }^{1-3}$

Masayo Takahashi ${ }^{1-3}$

Yasuo Kurimoto ${ }^{1-3}$

'Department of Ophthalmology, Kobe City Medical Center General Hospital, 2Department of Ophthalmology, Institute of Biomedical Research and Innovation Hospital, ${ }^{3}$ Laboratory for Retinal Regeneration, RIKEN Center for Developmental Biology, Kobe, ${ }^{4}$ Department of Ophthalmology, Kyoto University Graduate School of Medicine, Kyoto, Japan
Correspondence: Yasuhiko Hirami Department of Ophthalmology, Institute of Biomedical Research and Innovation Hospital, 2-2 Minatojima-minamimachi, Chuo-ku, Kobe, 650-0047, Japan Email yhirami@cdb.riken.jp
Background: The purpose of this study was to observe the characteristic findings of spectral-domain optical coherence tomography (SD-OCT) images in the retinas of patients with retinitis pigmentosa and to evaluate their distribution patterns in the early and advanced stages of the disease.

Methods: A total of 184 patients (368 eyes) with retinitis pigmentosa were observed using SDOCT. We studied the presence or absence of continuous inner/outer segment (IS/OS) lines, presence of thinning of the retinal pigment epithelium-Bruch's membrane complex, and distribution patterns of hyperreflective foci in the inner and outer nuclear layers (INL and ONL).

Results: The IS/OS junction had partially disappeared in 275 eyes, which were at the early stage of retinitis pigmentosa (group X), whereas the junction had totally disappeared in 93, which were at the advanced stage of retinitis pigmentosa (group Y). Hyperreflective foci in the INL were observed in a significantly larger proportion of the eyes in group $\mathrm{X}$ than in group $\mathrm{Y}(90 \%$ versus $61 \%, P<0.001)$, but hyperreflective foci in the ONL were observed in a significantly larger proportion of eyes in group $\mathrm{Y}$ than in group $\mathrm{X}(100 \%$ versus $69 \%, P<0.001)$.

Conclusion: Hyperreflective foci in the INL were more frequently observed in retinas with the early stage of retinitis pigmentosa and hyperreflective foci in the ONL were more frequently observed in the advanced stage. Hyperreflective foci may be indicative of changes in the retinal structure at each stage of retinitis pigmentosa.

Keywords: hyperreflective foci, spectral-domain optical coherence tomography, retinitis pigmentosa

\section{Introduction}

Retinitis pigmentosa is a heterogeneous group of hereditary degenerative retinal diseases characterized by primary apoptotic death of rod photoreceptors. ${ }^{1}$ Loss of rod photoreceptors initially causes night blindness and loss of the peripheral visual field and is followed by cone photoreceptor cell death, causing loss of central visual acuity in advanced cases. There are various patterns in the course of progression of retinitis pigmentosa, and these are believed to result from a variety of gene mutations that have been reported to be associated with the disease. More than 40 genes and ten loci are known to cause retinitis pigmentosa, most of which are associated with photoreceptor-specific functions such as phototransduction, maintenance of the unique cytoskeletal structure, intracellular signaling, and cell metabolism (http://www.sph. uth.tmc.edu/Retnet/).

Apoptotic photoreceptor cell death in retinitis pigmentosa is assumed to be induced by a lack of functional proteins or by intracellular accumulation of mutant proteins. ${ }^{2}$ 
Rod photoreceptor cell death is sometimes followed by degeneration of retinal pigment epithelium (RPE), and eventually leads to degeneration of cone photoreceptor cells. Because the histopathology of retinas with retinitis pigmentosa associated with specific gene mutations has recently been reported, the mechanisms of disease progression with some of the gene mutations have been partially elucidated. . $^{3,4}$ However, histopathological studies of autopsied eyes are still limited, as in most cases only the advanced stage of the disease has been observed.

Optical coherence tomography (OCT) is a method of noninvasively obtaining cross-sectional retinal images for the diagnosis and management of retinal diseases. Recently, spectral-domain (SD)-OCT retinal images with an axial resolution of 5-7 $\mu \mathrm{m}$ have been introduced into clinical practice. An SD-OCT instrument (Spectralis ${ }^{\circledR}$ OCT; Heidelberg Engineering, Heidelberg, Germany) enables high-speed acquisition of multiple B-scan images at the same location using a threedimensional eye-tracking system. Multiple B-scan averaging of these images facilitates high-quality data processing to reduced speckle noise and enables visualization of detailed retinal structures. ${ }^{5}$ Various structural changes in eyes with retinitis pigmentosa have been identified using SD-OCT.,

Hyperreflective foci (HFs) or small dense particles are observed in other pathological retinas, such as those with agerelated macular degeneration ${ }^{8}$ and diabetic retinopathy. ${ }^{9-11}$ The origin of these HFs has not been examined histologically. HFs have been considered as macrophages, migrating RPE cells, or extravasated lipoproteins resulting from retinal inflammation or the breakdown of retinal vessel barriers. In age-related macular degeneration, the amount of HFs is known to be reduced after anti-VEGF (vascular endothelial growth factor) therapy.

In this study, we aimed to investigate detailed changes in the retinas of patients with retinitis pigmentosa over the course of the disease using the Spectralis SD-OCT system. We examined 368 eyes from 184 patients with retinitis pigmentosa using multiple B-scan averaged images (reduced speckle noise) and observed various findings that were significant for understanding the patterns and processes of retinitis pigmentosa progression. We focused on HFs in SDOCT images and evaluated their distribution patterns in the early and advanced stages of the disease.

\section{Materials and methods}

We observed 368 eyes from 184 patients with retinitis pigmentosa in our outpatient clinic at Kobe City Medical Center General Hospital between January 2008 and May 2010.
Informed consent was obtained from all subjects (for patients under 20 years, patients' parental consent was also obtained); all procedures adhered to the Declaration of Helsinki and were approved by the institutional review board. All patients underwent a comprehensive ophthalmologic examination, including determination of best-corrected visual acuity and intraocular pressure, indirect ophthalmoscopy, noncontact lens slit-lamp biomicroscopy, a Goldmann kinetic perimetry test, and OCT examination. Electroretinogram tests were also performed for patients who had no history of such an examination prior to visiting our clinic.

The diagnosis of retinitis pigmentosa was made if patients had symptoms of night vision loss, visual field loss with ring scotoma or constricted visual fields detected by kinetic perimetry, retinal degeneration with typical bone spicule-like pigmentation of the fundus, and reduced amplitude determined by electroretinogram testing. Patients with crystalline retinopathy or Usher syndrome were excluded.

OCT images were obtained using the Spectralis OCT. To reduce the speckle noise, B-scan averaging of at least 40 cross-sectional retinal images obtained at the same location was performed using a three-dimensional eye-tracking system. We observed horizontal sections of the retinal images obtained by SD-OCT that included the fovea of both eyes of the subjects. When patients could not find the fixation light, the scan location was fitted manually to the slice with the foveal depression determined by the examiner. Patients for whom OCT images of both eyes were not obtained were excluded from the study. For example, in cases with very low visual acuity, it is difficult to obtain high-quality images because of poor eye fixation. We also excluded patients who had subfoveal cystoid macular edema, retinoschisis, epiretinal membrane, or vitreomacular traction syndrome, which may interfere with precise evaluation of the distribution of HFs.

The area of the remaining inner segment/outer segment (IS/OS) junction varied widely between subjects. We categorized all eyes into two groups based on the presence of a continuous IS/OS junction line at least $0.4 \mathrm{~mm}$ with no disruption which included the foveola, ie, the area with a diameter of $0.2 \mathrm{~mm}$ at the center of the fovea. ${ }^{12}$

In the OCT image of normal eyes (with reduced speckle noise), the boundaries of the retinal layers were well demarcated (Figure 1A). However, the images of eyes with retinitis pigmentosa showed several pathological findings, including different degrees of defects of the IS/OS junction and external limiting membrane reflex, thinning of the outer nuclear layer and of the reflex of the RPE-Bruch's membrane complex, and 
the presence of HFs in the inner nuclear layer, outer nuclear layer, and subretinal space (Figure 1B).

Statistical analyses for the frequency of eyes with HFs in the inner nuclear layer and outer nuclear layer within each group were performed using the chi-squared test and comparison of the average logMAR (logarithm of the minimum angle of resolution) visual acuity of each group was performed using the Student's $t$-test. If a $P$-value was less than 0.05 , the result was considered to be statistically significant.

\section{Results}

This study was a retrospective case series in which we reviewed the medical records of 368 eyes from 184 patients with retinitis
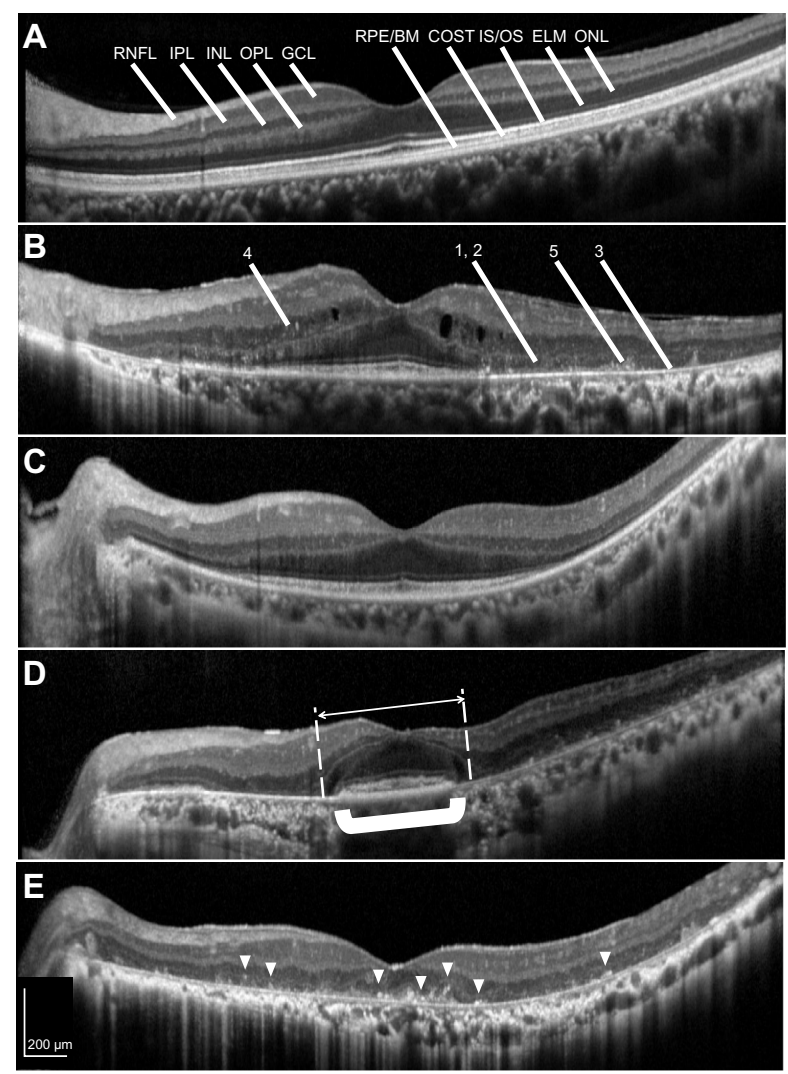

Figure I SD-OCT images of normal and RP retinas. (A) In the image of a normal eye (reduced speckle noise), the boundaries of the retinal layers are well demarcated. (B) In the image of an RP eye, some pathological findings were observed. (I) Defects of the IS/OS junction and ELM reflex, (2) thinning of the ONL, (3) thinning of the reflex of the RPE-Bruch's membrane complex, (4) HFs in the INL, and (5) HFs in the ONL and subretinal space. (C) RP retina without HFs in the ONL. In this eye, no thinning of the reflex of RPE-Bruch's membrane complex was observed. (D) In four cases, the ONL (white arrow) seemed to be preserved over a wider area than the RPE-Bruch's membrane complex (shown in bold bracket). (E) RP retinas with HFs (arrowheads) in the ONL. All RP eyes with no IS/OS under the fovea had HFs in the ONL.

Abbreviations: RNFL, retinal nerve fiber layer; IPL, inner plexiform layer; INL, inner nuclear layer; OPL, outer plexiform layer; GCL, ganglion cell layer; RPE/BM, retinal pigment epithelium and Bruch's membrane complex; COST, cone outer segment tips; IS/OS, inner segment/outer segment junction of photoreceptors; ELM, external limiting membrane; ONL, outer nuclear layer; SD-OCT, spectral-domain optical coherence tomography; RP, retinitis pigmentosa; HFs, hyperreflective foci. pigmentosa ( 82 male and 102 female). The average age of the patients was $52.0 \pm 16.0$ (range 14-84) years. The visual acuity of the eyes with retinitis pigmentosa ranged from hand motion to -0.18 in $\log$ MAR units (almost equal to 20/30).

The eyes were first categorized into two groups, ie, 275 of the 368 eyes had a continuous IS/OS junction line under the fovea (group X) while 93 eyes did not (group Y). Both eyes of all but three patients were categorized into the same group; the three remaining patients had one eye with a continuous IS/OS junction under the fovea and the other eye without an IS/OS junction.

In 191 eyes in group $\mathrm{X}$ (those with a continuous IS/OS junction under the fovea), we observed a variable degree of thinning of the RPE-Bruch's membrane complex. In these 191 eyes, we observed HFs in the outer nuclear layer, usually in the area where the IS/OS junction had disappeared (Figure 1B). On the other hand, in the remaining 84 eyes in group X, no thinning of the RPE-Bruch's membrane complex was observed within a $6 \mathrm{~mm}$ long area including the fovea, suggesting the presence of a healthy RPE extending over the area of the degenerating outer nuclear layer (Figure 1C). In contrast, in eight eyes of four cases in group $\mathrm{X}$, the outer nuclear layer seemed to be preserved over a wider area than the RPE-Bruch's membrane complex (Figure 1D). In group $\mathrm{Y}$, which showed no or disrupted IS/OS junctions, the RPE-Bruch's membrane complex thinning generally exceeded the area of the remaining IS/OS junction or outer nuclear layer, but varied widely in each case (Figure 1E).

We further focused on the distribution patterns of intraretinal HFs in the OCT images. HFs were sometimes found in normal eyes on the edge of the inner nuclear layer adjacent to the outer plexiform layer (Figure 2A and B). On the other hand, in the eyes with retinitis pigmentosa, $\mathrm{HFs}$ were typically found throughout the inner nuclear layer (Figure 2C and D). In group $\mathrm{X}$, the above-mentioned 84 eyes with healthy RPE over $6 \mathrm{~mm}$ long including the fovea had no HFs in the outer nuclear layer, although the IS/OS junction had disappeared within that area. In group Y, 93 eyes showed HFs in the outer nuclear layer with thinning of the outer nuclear layer and of the reflex of RPE-Bruch's membrane complex (Table 1). HFs in the outer nuclear layer were observed in a significantly larger proportion of eyes in group $\mathrm{Y}$ than in group $\mathrm{X}(100 \%$ versus $69 \%, P<0.001)$. HFs were also found in the inner nuclear layer of some eyes with retinitis pigmentosa, ie, $90 \%$ of eyes in group $\mathrm{X}$ and $61 \%$ of eyes in group Y. HFs in the inner nuclear layer were observed in a significantly larger proportion of eyes in group $\mathrm{X}$ than in group $\mathrm{Y}(P<0.001)$. HFs in the inner 


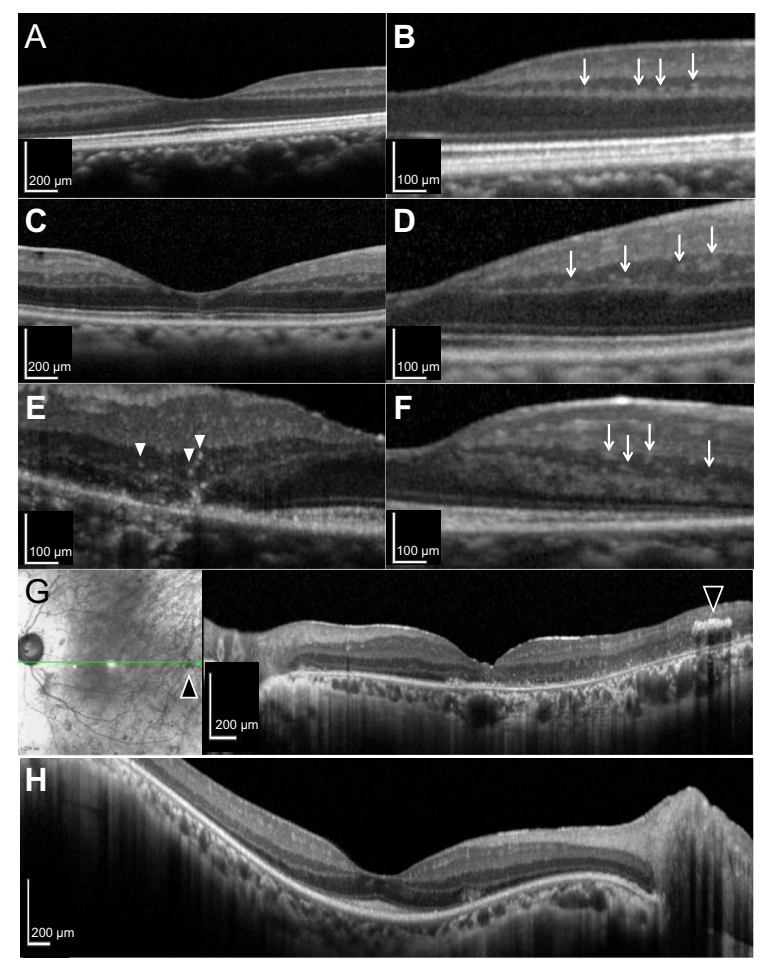

Figure 2 HFs in the INL of normal and RP retinas. (A and $\mathbf{B}$ ) HFs were located on the edge of the INL adjacent to the OPL in the normal retina (arrows). (C and D) HFs were located in the middle of the INL of the RP retina (arrows). HFs in the INL were of various sizes and signal intensities, ranging from relatively small and hyperreflective (E, arrowheads) to large and hyporeflective (F, arrows). (G) A highintensity object was observed in the INL by OCT in a location homologous to bone spicule-like pigmentation (arrowheads). $(\mathbf{H})$ In some cases with an IS/OS under the fovea, HFs were observed only in the ONL and not in the INL.

Abbreviations: HFs, hyperreflective foci; INL, inner nuclear layer; OPL, outer plexiform layer; IS/OS, inner segment/outer segment junction of photoreceptors; ONL, outer nuclear layer; RP, retinitis pigmentosa; OCT, optical coherence tomography.

nuclear layer varied in size and signal intensity, and ranged from relatively small and hyperreflective (Figure 2E) to large and hyporeflective (Figure 2F). HFs in the inner nuclear layer were often observed around the border where the IS/OS had disappeared or in the area with intact IS/OS lines,

Table I Number of eyes categorized by the presence or absence of a continuous IS/OS junction under the fovea and by location of $\mathrm{HFs}$ in the inner/outer nuclear layer (INL/ONL) of the retina

\begin{tabular}{|c|c|c|c|c|}
\hline \multirow[t]{2}{*}{ Location of HFs } & \multicolumn{2}{|c|}{$\begin{array}{l}\text { Group X (IS/OS } \\
\text { exists under fovea) }\end{array}$} & \multicolumn{2}{|c|}{$\begin{array}{l}\text { Group Y (IS/OS } \\
\text { absent under fovea) }\end{array}$} \\
\hline & $\mathrm{n}$ & $\begin{array}{l}\text { Average } \\
\text { BCVA } \\
\text { (logMAR) }\end{array}$ & $\mathbf{n}$ & $\begin{array}{l}\text { Average } \\
\text { BCVA } \\
\text { (logMAR) }\end{array}$ \\
\hline No & $8(3 \%)$ & 0.08 & 0 & \\
\hline INL only & 76 (28\%) & 0.05 & 0 & \\
\hline ONL only & $20(7 \%)$ & 0.22 & 36 (39\%) & 1.72 \\
\hline INL and ONL & $|7|(62 \%)$ & 0.35 & $57(61 \%)$ & 1.40 \\
\hline Total & 275 (100\%) & 0.24 & $93(100 \%)$ & 1.51 \\
\hline
\end{tabular}

Abbreviations: BCVA, best corrected visual acuity; HFs, hyperreflective foci; IS/ OS, inner segment/outer segment; logMAR, logarithm of the minimum angle of resolution; INL, inner nuclear layer; ONL, outer nuclear layer. and only rarely in the peripheral retina; in the latter case, they occurred along with advanced degeneration. We also observed high-intensity objects in the inner nuclear layer by OCT that were homologous with bone spicule-like pigmentation (Figure $2 \mathrm{G}$ ). In six eyes from three cases, we found HFs in the outer nuclear layer but not in the inner nuclear layer and without accompanying thinning of the reflex of RPE-Bruch's membrane complex (Figure 2H).

The average best-corrected visual acuity in each category is also shown in Table 1. The average visual acuity of eyes in group $\mathrm{X}$ was significantly better than that of eyes in group $\mathrm{Y}$ $(0.24$ versus $1.51, P<0.001)$. In group $\mathrm{X}$, the average visual acuity of eyes without HFs in the outer nuclear layer was significantly better than that of eyes with HFs in the outer nuclear layer ( 0.06 versus $0.33, P<0.001)$. The average visual acuity of eyes in group $X$ without any HFs showed no significant difference from the average visual acuity of eyes with HFs in the inner nuclear layer only ( 0.08 versus $0.05, P=0.16$ ).

\section{Discussion}

In this study, we observed retinal images from patients with retinitis pigmentosa obtained using SD-OCT and categorized these images based on the presence of the photoreceptor IS/OS junction and HFs in the outer and/or inner nuclear layer. SD-OCT enabled us to visualize detailed retinal structures noninvasively at every stage of the disease. Here, we focused on the specific changes observed on OCT in patients with retinitis pigmentosa.

Histopathological analysis of the retina in eyes with retinitis pigmentosa has revealed that the outer segments of rod photoreceptors are shortened and that this initial change in the degenerating retina is followed by gradual loss of whole cells as the disease progresses. ${ }^{13}$ Subsequently, RPE cells migrate into the inner retina and accumulate around the walls of retinal blood vessels. ${ }^{14}$

In the SD-OCT images of retinas with retinitis pigmentosa, disappearance of the IS/OS junction and the external limiting membrane may indicate damage to the IS and OS of photoreceptors. Thinning of the outer nuclear layer seems to be a result of photoreceptor cell death. We also observed thinning of the reflex of RPE-Bruch's membrane complex in areas in which the IS/OS junction had disappeared. Typically, in these areas, HFs were also observed in the outer nuclear layer. These HFs in the outer nuclear layer may be interpreted as resulting from photoreceptor cell death and subsequent RPE cell migration into the outer nuclear layer and degeneration. Bone spicules are typical features of the 
fundus in retinitis pigmentosa. These have already been examined in morphological studies and have been identified as translocated RPE cells. ${ }^{15}$ It may be difficult to prove that bone spicules and HFs constitute an identical population; it should be noted that HFs were more widely observed throughout the degenerating part of retina, whereas bone spicules were mainly found in the extrafoveal retina.

HFs in the inner nuclear layer were mainly observed in areas without thinning of the RPE-Bruch's membrane complex. This may suggest that HFs in the inner nuclear layer reflect some sort of change apart from that associated with RPE cells. Various changes have been reported, not only in the outer layers of the retina with retinitis pigmentosa but also in the inner layers, including reactive gliosis by Müller cells, migration of activated microglia, and expansion of the synaptic processes of photoreceptor cells, amacrine cells, and horizontal cells in the course of synaptic remodeling; this is known as neurite sprouting in both human and animal models. ${ }^{15-18} \mathrm{HFs}$ in the inner nuclear layer may reflect such changes.

HFs in the outer nuclear layer were observed in a significantly larger proportion of group $\mathrm{Y}$ eyes with an advanced stage of retinitis pigmentosa in which the outer retina had already been destroyed, compared with group $\mathrm{X}$ eyes. In contrast, HFs in the inner nuclear layer were observed in a significantly larger proportion of group $\mathrm{X}$ eyes than in group $\mathrm{Y}$ eyes. Intraretinal changes accompanying HFs in the inner nuclear layer may occur in the early stage of retinitis pigmentosa. Eight eyes in group $\mathrm{X}$ had no HFs in the inner or outer nuclear layers, and these patients were young (aged 21-28 years), and we speculated that these cases were in the very early stages of the disease.

Visual acuity was preserved in the eyes without HFs in the outer nuclear layer, which was considered as the early stage of disease. Eight eyes in group $\mathrm{X}$ without any HFs showed worse visual acuity than 76 eyes with HFs in the inner nuclear layer only, but the difference was not statistically significant.

Based on these observations, we hypothesized that the patterns of distribution of HFs in the SD-OCT images are associated with disease progression of typical eyes with retinitis pigmentosa as follows (Figure 3): eyes with HFs only in the inner nuclear layer but not in the outer nuclear layer may represent the early stage of degeneration, and remodeling of neural circuits in the inner nuclear layer during the decay of rod photoreceptors; HFs in the inner nuclear layer were often observed around the border of the area where the IS/OS disappeared or in areas with intact IS/OS lines, and only rarely in the peripheral retina, which is in the advanced stage of degeneration. In the more advanced stages of the disease, degeneration of the RPE leads to migration of RPE cells into the outer retina and these are detected as HFs in the outer nuclear layer. In extremely advanced cases, all retinal layers are thinned and there is no further remodeling of the inner retinal layers. At this stage of the disease, HFs in the thinner nuclear layer may no more be distinct.

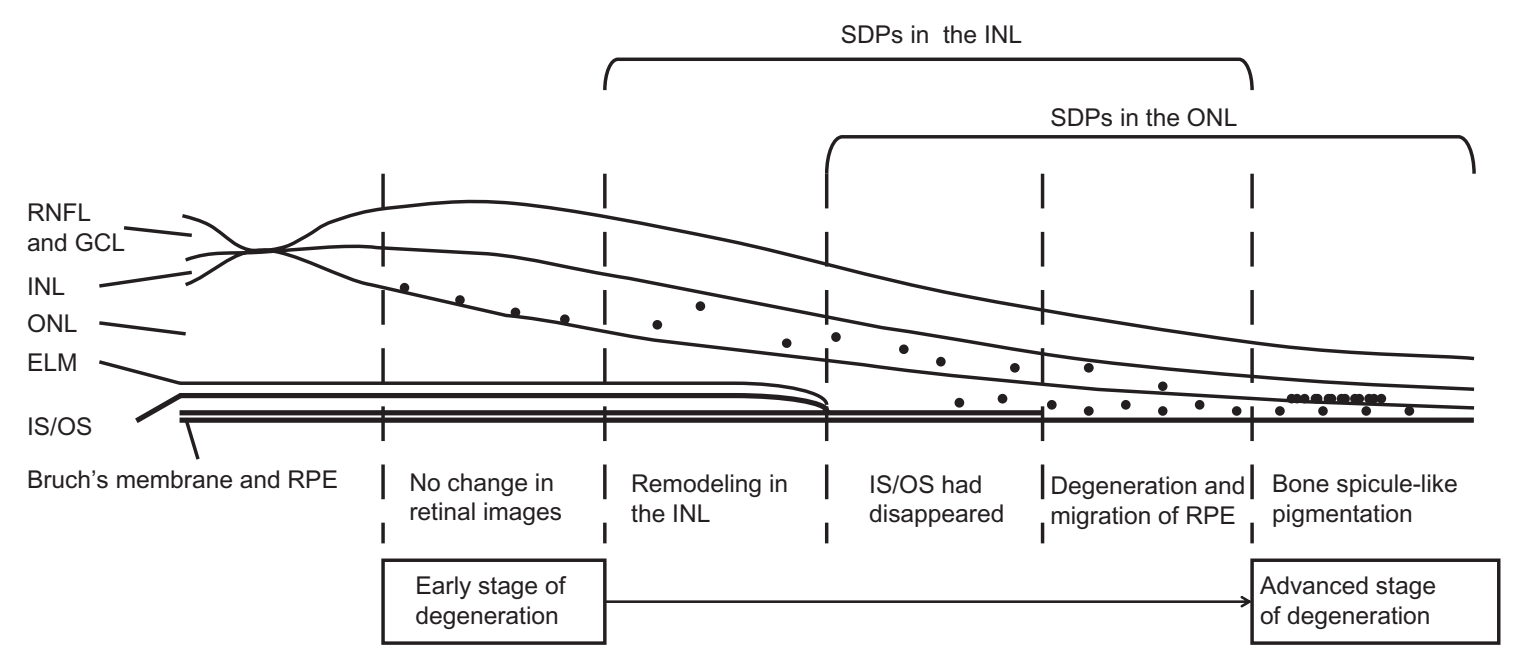

Figure 3 Hypothetical association between HFs observed on SD-OCT images and disease progression in typical RP retinas. Eyes with HFs in the INL but not in the ONL may be in the early stage of degeneration, and remodeling of neural circuits in the INL occurs after the decay of rod photoreceptors. HFs in the INL were often observed around the border of the area where the IS/OS disappeared or in areas with intact IS/OS lines, and only rarely in the peripheral retina, the latter of which was indicative of advanced degeneration. In the more advanced stage of disease, RPE degeneration leads to migration of RPE cells into the outer retina and these are observed as HFs in the ONL. In extremely advanced cases, all retinal layers are thinned and there is no further remodeling of the inner retinal layers. In this stage of the disease, HFs in the INL may not be distinct.

Abbreviations: RNFL, retinal nerve fiber layer; INL, inner nuclear layer; GCL, ganglion cell layer; RPE, retinal pigment epithelium; IS/OS, inner segment/outer segment junction of photoreceptors; ELM, external limiting membrane; ONL, outer nuclear layer; SD-OCT, spectral-domain optical coherence tomography; SDPs, small dense particles; RP, retinitis pigmentosa; HFs, hyperreflective foci. 
Retinitis pigmentosa is a collective designation of heterogeneous degenerative diseases. The clinical features associated with each type of genetic background have not been individually investigated as yet, but OCT images should prove helpful for categorizing clinical entities in more detail and correlating these with genetic information or hereditary patterns. However, in six eyes of three cases, we found HFs in the outer nuclear layer but not in the inner nuclear layer, without accompanying thinning of the reflex of RPE-Bruch's membrane complex. We do not have an explanation for these cases, but it is possible that they constitute a specific type of retinitis pigmentosa in which degeneration of the outer retina progresses without remodeling of the inner retina or RPE degeneration. In eight eyes of four cases, the outer nuclear layer seemed to be preserved over a wider area than the RPEBruch's membrane complex. The finding seemed to be of a hyporeflective wedge-shaped band, which has been reported in eyes with geographic atrophy secondary to age-related macular degeneration. In these cases, the RPE degeneration around the macular area might have happened before the degeneration of photoreceptor cells. ${ }^{19}$ Further studies are needed to follow time-dependent changes in each case and to correlate genetic mutations and observation of OCT images. These will provide an insight into the pathology of retinitis pigmentosa according to genetic background and information for predicting the prognosis of each patient.

\section{Disclosure}

The authors report no conflicts of interest in this work.

\section{References}

1. Weleber RG, Gregory-Evans K. Retinitis pigmentosa and allied disorders. In: Ryan SJ, editor. Retina. 4th ed. Amsterdam, The Netherlands: Elsevier; 2006.

2. Li ZY, Wong F, Chang JH, et al. Rhodopsin transgenic pigs as a model for human retinitis pigmentosa. Invest Ophthalmol Vis Sci. 1998;39: 808-819.

3. Jacobson SG, Cideciyan AV, Aleman TS, et al. Crumbs homolog 1 (CRB1) mutations result in a thick human retina with abnormal lamination. Hum Mol Genet. 2003;12:1073-1078.
4. To K, Adamian M, Berson EL. Histologic study of retinitis pigmentosa due to a mutation in the RP13 gene (PRPC8): comparison with rhodopsin Pro23His, Cys110Arg, and Glu181Lys. Am J Ophthalmol. 2004;137:946-948.

5. Hangai M, Yamamoto M, Sakamoto A, Yoshimura N. Ultrahighresolution versus speckle noise-reduction in spectral-domain optical coherence tomography. Opt Express. 2009;17:4221-4235.

6. Mitamura Y, Mitamura-Aizawa S, Nagasawa T, Katome T, Eguchi H, Naito T. Diagnostic imaging in patients with retinitis pigmentosa. J Med Invest. 2012;59:1-11.

7. Triolo G, Pierro L, Parodi MB, et al. Spectral domain optical coherence tomography findings in patients with retinitis pigmentosa. Ophthalmic Res. 2013;50:160-164.

8. Framme C, Wolf S, Wolf-Schnurrbusch U. Small dense particles in the retina observable by spectral-domain optical coherence tomography in age-related macular degeneration. Invest Ophthalmol Vis Sci. 2010;51: 5965-5969.

9. Ota M, Nishijima K, Sakamoto A, et al. Optical coherence tomographic evaluation of foveal hard exudates in patients with diabetic maculopathy accompanying macular detachment. Ophthalmology. 2010;117: 1996-2002.

10. Framme C, Schweizer P, Imesch M, Wolf S, Wolf-Schnurrbusch U. Behavior of SD-OCT-detected hyperreflective foci in the retina of anti-VEGF-treated patients with diabetic macular edema. Invest Ophthalmol Vis Sci. 2012;53:5814-5818.

11. Bolz M, Schmidt-Erfurth U, Deak G, Mylonas G, Kriechbaum K, Scholda C; Diabetic Retinopathy Research Group Vienna. Optical coherence tomographic hyperreflective foci: a morphologic sign of lipid extravasation in diabetic macular edema. Ophthalmology. 2009;116: 914-920.

12. Yamada E. Some structural features of fovea centralis in the human retina. Arch Ophthalmol. 1969;82:151-159.

13. Milam AH, Li ZY, Cideciyan AV, Jacobson SG. Clinicopathologic effects of the Q64ter rhodopsin mutation in retinitis pigmentosa. Invest Ophthalmol Vis Sci. 1996;37:753-765.

14. Li ZY, Possin DE, Milam AH. Histopathology of bone spicule pigmentation in retinitis pigmentosa. Ophthalmology. 1995;102: 805-816.

15. Fariss RN, Li ZY, Milam AH. Abnormalities in rod photoreceptors, amacrine cells, and horizontal cells in human retinas with retinitis pigmentosa. Am J Ophthalmol. 2000;129:215-223.

16. Gupta N, Brown KE, Milam AH. Activated microglia in human retinitis pigmentosa, late-onset retinal degeneration, and age-related macular degeneration. Exp Eye Res. 2003;76:463-471.

17. Marc RE, Jones BW, Watt CB, et al. Neural remodeling in retinal degeneration. Prog Retin Eye Res. 2003;22:607-655.

18. Li ZY, Kljavin IJ, Milam AH. Rod photoreceptor neurite sprouting in retinitis pigmentosa. J Neurosci. 1995;15:5429-5438.

19. Mones J, Biarnes M, Trindade F. Hyporeflective wedge-shaped band in geographic atrophy secondary to age-related macular degeneration: an underreported finding. Ophthalmology. 2012;119:1412-1419.
Clinical Ophthalmology

\section{Publish your work in this journal}

Clinical Ophthalmology is an international, peer-reviewed journal covering all subspecialties within ophthalmology. Key topics include: Optometry; Visual science; Pharmacology and drug therapy in eye diseases; Basic Sciences; Primary and Secondary eye care; Patient Safety and Quality of Care Improvements. This journal is indexed on Submit your manuscript here: http://www.dovepress.com/clinical-ophthalmology-journal

\section{Dovepress}

PubMed Central and CAS, and is the official journal of The Society of Clinical Ophthalmology (SCO). The manuscript management system is completely online and includes a very quick and fair peer-review system, which is all easy to use. Visit http://www.dovepress.com/ testimonials.php to read real quotes from published authors. 